\title{
New Design Method for Lap Scarf Joint Used for Reconstruction of Timber Structures
}

\author{
Hana Hasníková, Jiř́i Kunecký, Michal Kloiber, Václav Sebera, Jan Tippner
}

\begin{abstract}
The article describes a development of a new carpentry join suitable for reconstructions of timber structures; it is possible to replace only damaged parts of wooden beam with help of the joint. The mechanical behavior of the joint was analyzed in details. The inclined faces transfer the direction of loading and use of less stiff wooden dowels allows better distribution of stresses. The design methodic has been created so that the maximum of joint potential is used safely.
\end{abstract}

Keywords Lap scarf joint, wooden dowels, stiffness, reconstruction, design rules

\section{Introduction}

Each country should take care of its culture heritage, which helps to preserve the identity of the nation. Historical buildings are part of the immovable heritage and a reconstruction of a high quality allows preserving them for future generations. In Central Europe there are many old timber structures that have to be taken care of. The aim of the research supported by Czech Ministry of Culture was a creation of fully-fledged base for designing of all-wooden carpentry joints suitable for sensitive reconstructions of historical timber structures.

The lack of maintenance and high moisture content often lead to material degradation. The level and extent of degradation can be assessed by various diagnostic methods, both non-destructive [1] and semi-destructive [2]. The ends of timber beams from roof trusses lying on the wet masonry are the typical example. To preserve as much original historically valuable wood as possible, the ideal repair consists in replacing only necessary amount of decayed wood by new material, see Fig. 1. New parts should be of same shape, dimensions and wood specie. Also, the physical and mechanical properties should be compatible with the existing structure. Nowadays, the steel pins are used for prosthesis connection. The design is supported by information in EC 5 (European Design Standard for Timber Structures) [3]. Steel pins are stiff and bearable, the design is relatively easy, but an interface of two different materials (steel $\times$ wood) in the joint may cause negative interaction such as considerable embedment zone or condensation of water vapor.

Using all-wooden carpentry joint for reconstruction of valuable timber structures is more adequate for respecting

Hana Hasníková, Jiří Kunecký, Michal Kloiber

Institute of Theoretical and Applied Mechanics, v. v. i., Czech Academy of Sciences

Czech Republic

Václav Sebera, Jan Tippner

Department of Wood Science, Mendel University in Brno

Czech Republic
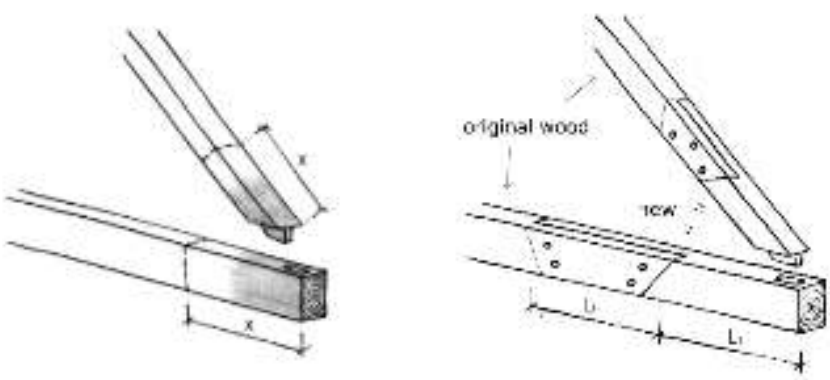

Figure 1. The idea of replacement; the damaged wood of an original old timber beam (grey part) is cut off and replaced with the help of a joint

the aesthetical and historical character of the structure. It is conspicuous and its manufacturing by a skilled craftsman helps to keep the awareness of the traditional craft as a part of cultural heritage. However, such a joint has to fulfil strict requirements of mechanical behavior; it has to be stiff and resistant enough.

Roof trusses are complicated structures and their structural members can be loaded differently according to the role in the system. Therefore it is advantageous to use a joint that can work sufficiently under various loading situations - it should carry tension, compression, bending moment and shear. Such a connection is introduced in the article. Four variants of the joint are presented below, see Fig. 2.

\section{Research}

\section{A. Joint behaviour}

Forces in carpentry joints are transfered by compression and friction on the contact surfaces of the jointed parts. Timber connectors (dowels and pegs) are used for the force transfer in the presented joint as well, contrary to the dowels
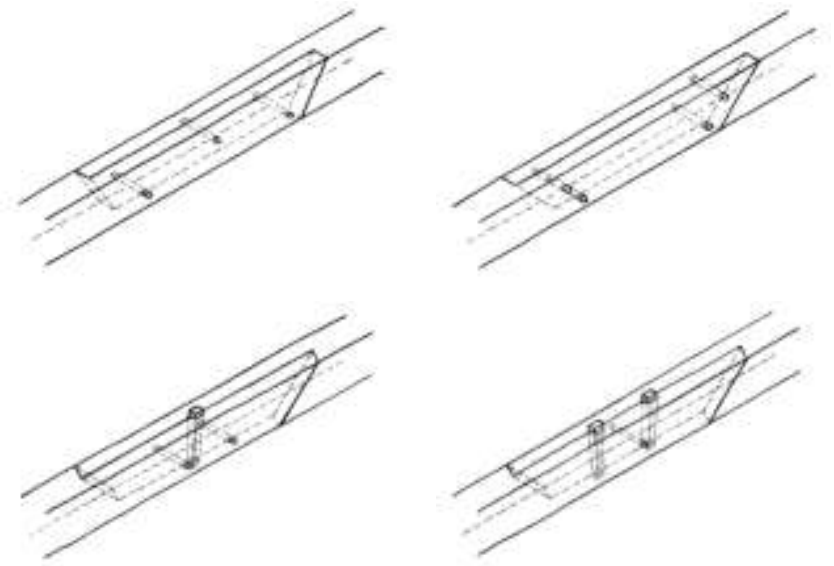

Figure 2. Four variants of all-wooden lap joint with oblique faces; three dowel joint (top left), four dowel joint (top right), one peg joint with two dowels (bottom left) and two peg joint with one dowel 
in e.g. tenons or dovetail joints where they help to ensure the correct position of the jointed members.

Joints that carry bending moment are designed with difficulties because of lack of information about force distribution in the connection. The use of oblique faces and the wooden dowels only is innovative in the lap scarf joint design. During the research which focused also on optimization of joint parameters (length of joint, the angle of faces, number of dowels etc.) the mechanical behavior of the joint was analyzed in details.

Participation of oblique faces in mechanical behaviour of the joint is the basic principle. The faces carry the shear force, and thereby they reduce the forces acting on the dowels perpendicular to the timber grains (less resistant direction of the material), see Fig. 3. At the same time, the force direction in the joint and the dowels is changed from perpendicular to paralel to the grains (more resistant) by faces strutting while beam bending. The timber connectors (hardwood) are less stiff than steel ones. Their stiffness is more similar to the stiffness of the joined material (usually softwood), therefore even stress distribution in the embedment zone is observed. The joint has relatively small amount of connectors, because its stiffness has to be balanced. Too many dowels cause too stiff joint and the repaired beam colapses in the reduced cross section. Also, a large diameter of a timber dowel means high stiffness and high carrying capacity of the connector and does not allow oblique faces to transfer as much force as it can. The contact length of the faces reacts to the position of the joint within the beam. Placing joint as close to the support as possible (according to the condition of the original material) is the most advantageous design solution. The length of the joint influences the load-bearing capacity. The development of the joint can be sketched out in sequence of works of Fajman et al. [4, 5] who studied forces acting in the joint using mathematical model.

\section{B. Experimental testing}

Experimental and numerical analyses were performed to investigate parameters such as stiffness and strength of this type of prosthesis. The joint was tested under various loading conditions that simulate the real actions in the timber structure, especially in the historical roof trusses, i.e. pure bending (ceiling beams), combination of bending and compression (rafters) and bending and tension (tie beam).
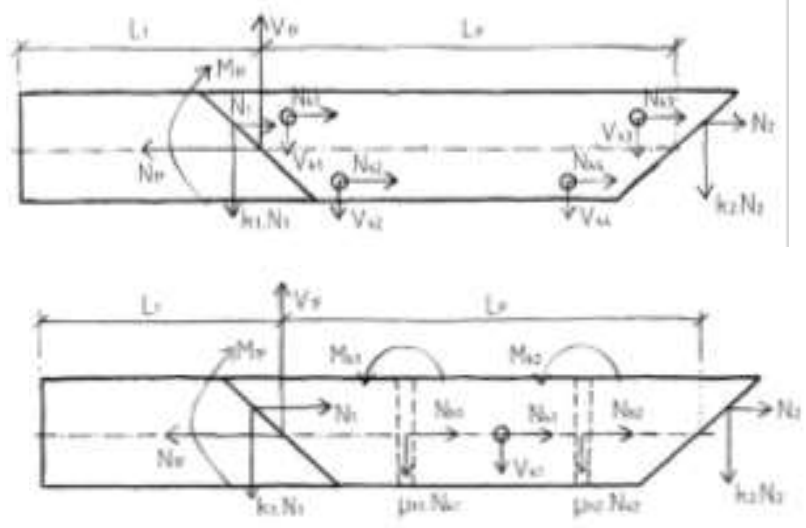

Figure 3. Distribution of forces in a four-dowel joint with oblique faces (top) and joint with two vertical wooden pegs and one dowel (bottom)

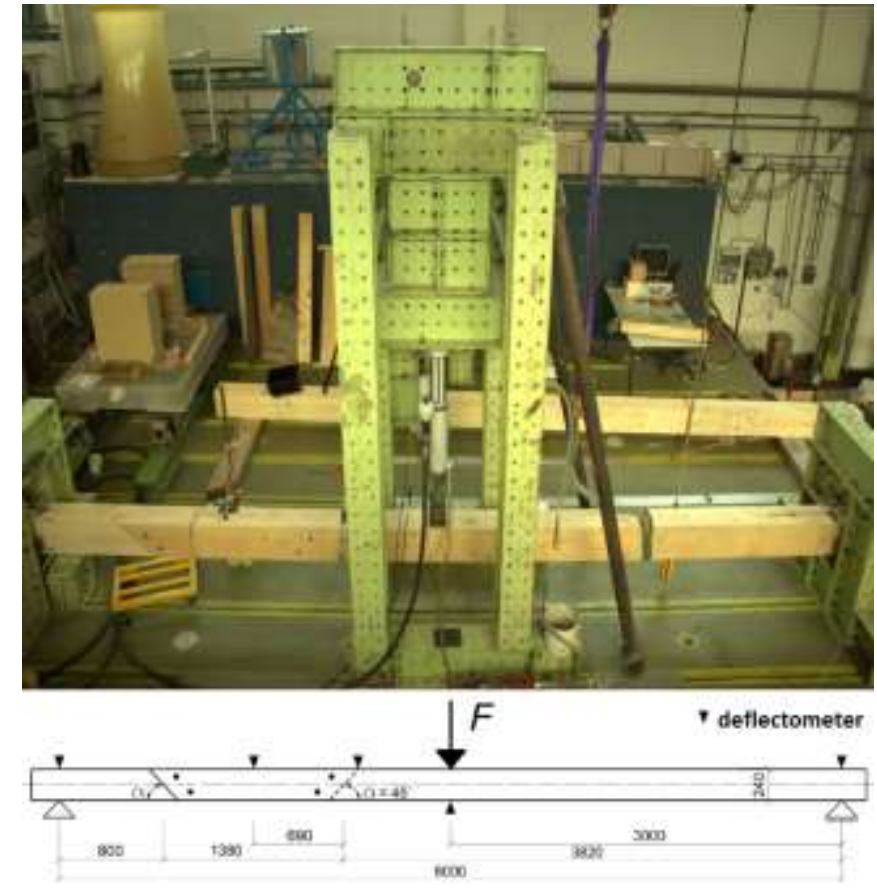

Figure 4. Experimental testing set-up; three-point bending test of a beam with a joint in scale $1: 1$

Experimental work has been designed for testing the jointed beams in three scales; the lap joint itself was tested in several variations differing in number and kind of timber connectors. The specimens were made of spruce and the dowels and pegs were made of oak wood. The samples were manufactured by a carpenter who specializes in traditional technology of woodworking and uses copies of original tools. Moisture content was always measured and taken into account while evaluation as it influences the timber behaviour. Material properties of all the samples were analyzed after testing also.

The three-point bending test set-up was used for the most of experiments, the joint was loaded by bending moment and shear force, see Fig. 4. Samples with the dimension $200 \times 240 \times 6000 \mathrm{~mm}$ were tested in pure bending; smaller samples $(100 \times 120 \times 3000 \mathrm{~mm}$, $50 \times 60 \times 1500 \mathrm{~mm}$ ) allowed set-ups for testing under combined loading. The reference beams without the joint or beams with joints with steel connectors were tested, so that the results with all-wooden joints can be compared. Many developmental variants of the joint were prepared joint with only one dowel or even none dowel, joints with different width of laps, joints in different positions within the beam etc. The tests of single timber connectors were made as well. DIC method (Digital Image Correlation) was applied succesfully for observing the behaviour of the oblique faces and determining the contact area. Interesting parts of experimental work has been already described by Kunecký et al. [6, 7].

\section{Numerical modeling}

Finally, a finite element model was constructed in ANSYS software and validated using experimental output, see Fig. 5. The element type SOLID95 was used in the model and contact between the joint faces and between holes in joint and dowels was defined as the standard contact 


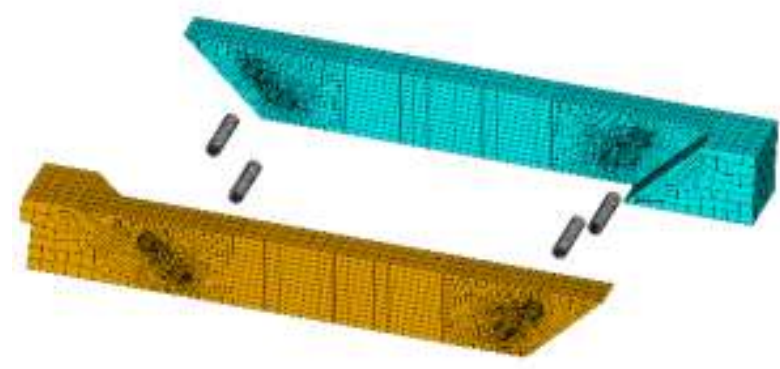

Figure 5. Numerical model of four-dowel joint in ANSYS software

using 3D surface-to-surface contact elements CONTA174 and target elements TARGE170. Two types of wood materials were defined in each FE model: a) material of beam made of Norway spruce, and b) material of dowel made of oak. Both wooden materials were modeled as linear elastic orthotropic materials. The models were used for estimating the stiffness of the beams with the joint and for extending the portfolio of tested dimensions of beams and connectors. Various combinations, that could not be tested physically, were simulated (e.g. small cross section with dowels larger than recommended). The reasonable intervals of dowel diameter for specific beam cross section were determined. Numerical models of the joint has been described by Arciszewska-Kedzior et al. [8].

The experimental results verified both the analytical mathematical model and numerical model and were used to determine the failure criteria (strength of timber, carrying capacity of dowel/hole system in various directions, carrying capacity of the obligue face, the dowel or the peg). All the criteria were related to the elastic limit. The diagrams of load-bearing capacity $M-N$ for ultimate limit state design and both stiffness and deflection relationships for serviceability limit state design were derived from the models.

\section{Practical Use}

\section{A. The methodic}

The methodic for designing all-wooden lap joints for reconstruction of historical structures was created on bases of previously described research. It focuses mainly on designers, structural engineers and building companies to help them use the joint in the reconstruction projects. It can be also useful for preservationists. Besides the design and calculation itself, it contains a chapter about manufacturing the joint and maintenance.

At the beginning of design, the information from site are important and define input data. The cross section and the span $L$ of the repaired structural member is known and quality of the original timber is assessed. The location of the joint within the beam $L_{l}$ can be adjusted, but is moreless given also. While designing the joint following parameters, see Fig. 6, have to be decided: length of the joint $L_{p}$, angle of the faces $\alpha$ and the kind of the joint. Four variants of the joint are presented in the methodic. Each of them has its advantages such as easier and faster manufacturing process (three dowel joint) or higher load-bearing capacity (two peg joint with one dowel) and an ideal way of use according to

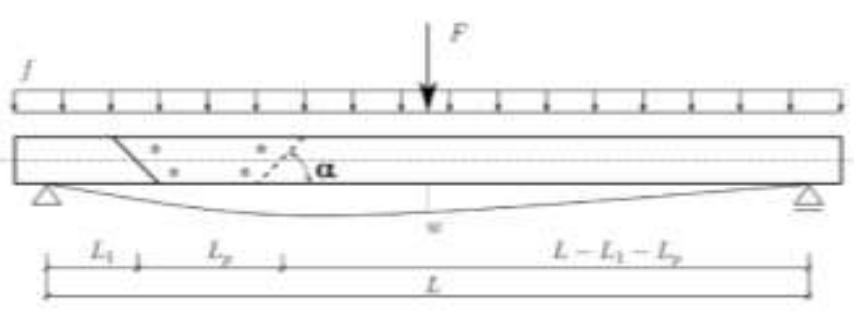

Figure 6. The design parameters of the beam with the joint

the loading of the repaired structural member. There are detailed drawings with recommended dimensions of connectors for all four of them in the methodic.

The engineer or a designer using the methodic has to understand mechanics so that he can apply the diagrams and equations correctly. He is responsible for the design and the safety level.

\section{B. Ultimate limit state}

The engineer has to estimate the loads acting on the structure and calculate the major inner forces, bending moment $M$ and normal force $N$, loading the individual member to be repaired. The values are then compared with the design diagrams $M-N$ of the certain cross section, see Fig. 7. There are two limit borders in the diagram corresponding to the length of the joint $L_{p}$. Short version equal to $2,5 h$ was inspired by minimal distances between connectors given by EC5, longer version equal to $5 h$ is limited by manufacturing and preservation point of view ( $h$ is the height of the cross section). It is possible to interpolate between two borders as the designer may need the joint with the length $L_{p}$ of $4 h$ for instance. In the Fig. 7 there are three points representing several cases that may occur while designing. Point no. 1 represents a ceiling beam loaded mainly by bending moment, length of the joint has to be more than 2,5h. Point no. 2 represents a tie beam highly loaded in tension that can not be repaired with help of the certain version of the lap joint, it would fail. The two peg joint could used instead as it has higher load bearing capacity. Point no. 3 represents the structural member such as rafter loaded by combination of bending and compression. The joint with the minimal length $L_{p}$ equal to $2,5 h$ is safe to use.

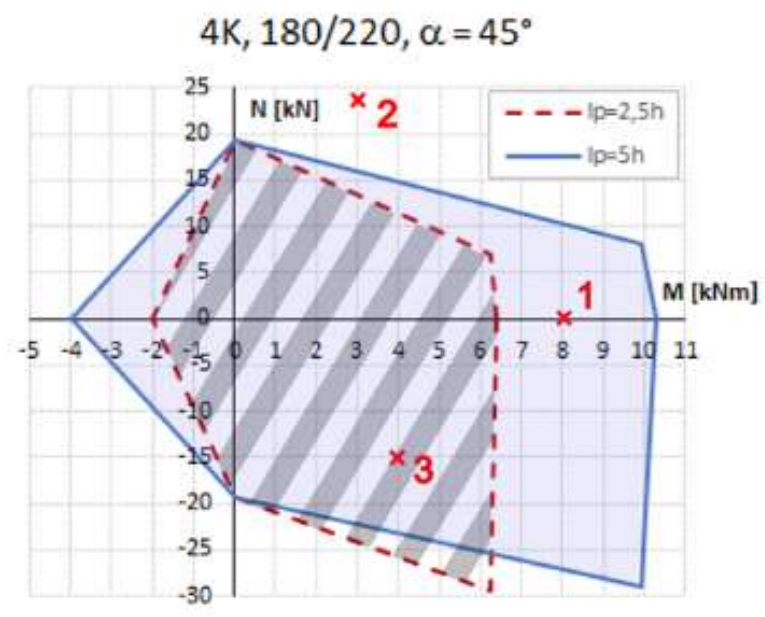

Figure 7. Example of a design diagram of load-bearing capacity of four dowel joint $4 \mathrm{~K}$ with cross section of $180 \times 220 \mathrm{~mm}$, angle of the faces is $45^{\circ}$ 
There are diagrams for several cross sections in the methodic that are the most common in the (roof) structures. It is possible to interpolate to get the missing cross section dimension. Three examples of design are presented in the text to help engineers understand the methodic even better.

\section{Serviceability limit state}

For each joint there is an equation for calculating the stiffness $k$ of the beam with the particular joint in the methodic. A deflection of the structural member is another criterion for design and the deflection in the middle of span $w$ of the beam with a joint is presented also. The engineer needs several parameters for the calculation; the example of the relationships for the beam with four dowel joint is shown below in (1) and (2). Position of the joint $L_{l}$ has to be less than $L / 12$, the length of the joint $L_{p}$ is $2,5 h$.

$$
\begin{gathered}
k=870 E b h^{2,6} / L^{2,6}\left[\mathrm{MNm}^{-1}\right] \\
w=w_{0} 4,6 h^{0,4} / L^{0,4} \quad[\mathrm{~m}]
\end{gathered}
$$

The parameters in the equation are following: $b$ is the width of the cross section $[\mathrm{m}], h$ is the height of the cross section $[\mathrm{m}], L$ is the span of the structural member $[\mathrm{m}], E$ is the average value of Young modulus $[\mathrm{GPa}]$ and $w_{0}$ is the deflection of the beam without the joint $[\mathrm{m}]$.

\section{Reconstruction}

The pilot projects of reconstruction with use of the presented joint has been completed, see Fig. 8. There are some practical notes mentioned in the methodic, that especially building companies have to focus on while reconstruction.

The knots in the material can influence the behaviour of the joint, therefore they should not occur close to the dowels or obligue faces. Timber of a high quality for both the prosthesis and the connectors has to be choosen. The drying cracks can reduce the load bearing capacity of the joint and the beam itself, no connector should be placed there. The drying of the timber will occur while the life of the repaired structure, it has to be taken into account while reconstruction. The manufacturing of the joint has to be precise. The maintenance not only of the joint but also of the whole structure is necessary for them to survive in a good condition as long as posible.

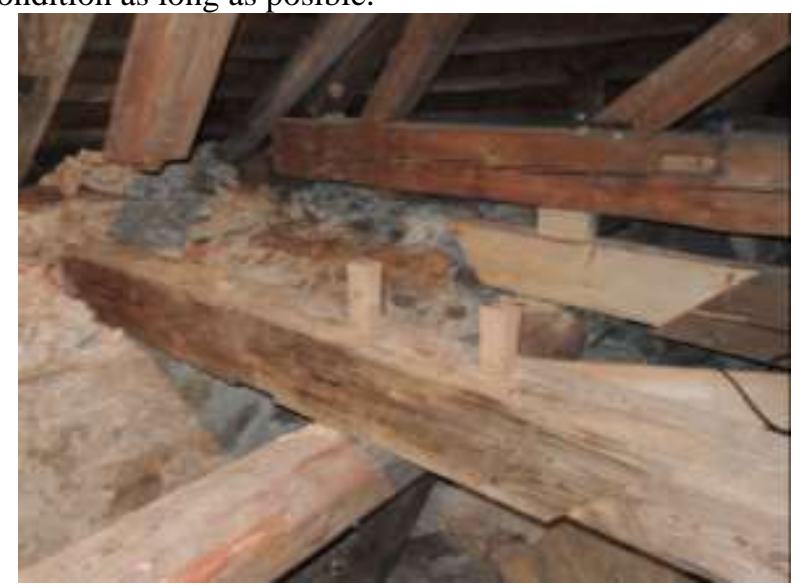

Figure 8. Reconstruction of a timber roof structure of castle in Lanškroun with help of two peg lap joint with obligue face

\section{Conclussion}

Half lap scarf joint with obligue faces and wooden connectors was presented and mechanical principles that allows the joint to transmit forces were clarified. The design methodic and application handbook have been created so that joint is used safely and efficiently. Authors are working on translation into English so that the methodics can be used not just in the Czech Republic in the future.

\section{Acknowledgment}

The authors would like to thank to project NAKI DF12P01OVV004, „Design and Assessment of Timber Joints of Historical Structures" provided by the Ministry of Culture of the Czech Republic for financial support.

\section{References}

[1] J. Pošta, J. Dolejš, "Non-Destructive Assessment of Timber Elements with an Emphasis on Radiometry", Int. J. Archit. Herit., vol. 9, is. 6, pp. 655-664, 2015.

[2] M. Kloiber, J. Tippner, J. Hrivnák, "Mechanical properties of wood examined by semi-destructive devices", Mater. Struct., vol. 78, is. 1-2, pp. 199-212, 2015. ISSN 1359-5997.

[3] EN 1995-1-1 Eurocode 5: Design of Timber Structures - Part 1-1: General - Common rules and rules for buildings. 2006.

[4] P. Fajman, J. Máca and J. Kunecký, "Joints in historical timber structures", Proceedings of Structural Faults and Repair 2014, p. 8, 2014.

[5] P. Fajman, J. Máca, "Scarf joints with pins or keys and dovetails", Proceedings of SHATIS'15 Structural Health Assessment of Timber Structures, pp. 899-906, 2015.

[6] J. Kunecký, V. Sebera, H. Hasníková, A. Arciszewska-Kedzior, J. Tippner, M. Kloiber, "Experimental assessment of a full-scale lap scarf timber joint accompanied by a finite element analysis and digital image correlation", Constr. Build. Mater., vol. 76, pp. 24-33, 2015. ISSN 0950-0618.

[7] A. Arciszewska-Kedzior, J. Kunecký, H. Hasníková, "Mechanical response of a lap scarf joint with inclined faces and wooden dowels under combined loading”, Proceedings of SHATIS'15 Structural Health Assessment of Timber Structures, pp. 849-858, 2015.

[8] A. Arciszewska-Kedzior, J. Kunecký, H. Hasníková, V. Sebera, "Lapped scarf joint with inclined faces and wooden dowels: experimental and numerical analysis", Eng. Struct., vol. 94, pp. 1-8, 2015. ISSN 0141-0296.

The all-wooden lap scarf joint is an ideal solution how to preserve the original material in a valuable historical timber structure. 